Method Small cycles of change included uploading updated plans to the local intranet portal, educating staff on how to find the plans using a 'how to' guide, education and posters placed around the wards. Effectiveness of the change was evaluated by time taken by medical staff to find a PAC-Plan using a hospital identification number.

Results After 3 small cycles of change there was a fall of $83 \%$ in median time taken to access the PAC-Plan from 4 minutes 55 seconds to 50 seconds.

Conclusion A significant reduction in time taken to find PACPlans using the existing intranet system was achieved through a combination of correctly uploading PAC-Plans, and training staff. Further work would be to ensure this system is sustained.

By ensuring appropriate logins and access to the NHS portal, any member of the palliative care team will be able to securely access PAC-Plans from any location across the region.

\section{G89(P) IMPROVING PRIMARY CARE ASSESSMENT OF FEVER IN CHILDREN UNDER FIVE}

${ }^{1} S$ Huang, ${ }^{2} \mathrm{~A}$ Hassan. ${ }^{1}$ Neonatalogy, London North West University Healthcare NHS Trust, London, UK; ${ }^{2}$ General Internal Medicine, Royal Bolton NHS FT, Bolton, UK

\subsection{6/archdischild-2020-rcpch.70}

Aim In a busy GP surgery with 4897 registered patients, this project aimed to review and improve the assessment and management of pyrexia in children under five. As recommended in NICE's guidance, all children presenting with a feverish illness should have their temperature, heart rate, respiratory rate and capillary re-fill time assessed and recorded.

Methods Using the Plan-Do-Study-Act (PDSA) quality improvement methodology, we reviewed consultation notes of all children under five to identify relevant cases and assess our performance. We designed a 'Fever $<5$ ' SystmOne template that allowed clinicians to complete prompts for vital signs when assessing relevant patients. We presented our findings and template at a practice meeting and reviewed consultation notes for relevant patients to assess whether our performance has improved in an one month period.

Results Our practice achieved $100 \%$ on the measurement and recording of the vital signs recommended by NICE when assessing children under five with recorded tympanic temperatures of 38 degrees or above; however, there was room for improvement. When assessing children under five presenting with a history of fever, the vital signs were only recorded $46 \%$ of the time. After the template was introduced and results shared at a practice meeting, we re-audited relevant patients in a one month period. This time, the four vital signs were measured and recorded $85 \%$ of the time.

Conclusion Feverish illness is a cause of parental concern. Not only is pyrexia one of the commonest reasons for a child to be taken to a doctor, it is often indicative of underlying infections, which remain the leading cause of death in children under five. As such, comprehensive assessment in primary care is vital. As pyrexia can often be variable in children, and parents often give paracetamol or ibuprofen prior to attending the surgery, it is important to include children with a history of fever in this population. With the introduction of a succinct and simple electronic template, we hope to improve our practice's assessment of children under five presenting with fever.
G90(P) MANAGING ACUTE PAEDIATRIC PAIN: A JOINED UP APPROACH

R King, C Hollingsworth. Paediatrics, Dorset County Hospital, Dorchester, UK

\subsection{6/archdischild-2020-rcpch.71}

Objectives Managing pain in children presenting to hospital should be a high priority for staff in the emergency department (ED) and on the children's ward. Many factors influence the quality of this provision. Following an audit of pain management in children with fractures, the Royal College of Emergency Medicine (RCEM) identified potential improvements in the care of children presenting to District General Hospitals having sustained a fracture. As part of a department wide focus on pain management and closer links being forged between paediatrics and $\mathrm{ED}$, this project aimed to assess staff confidence in paediatric pain management as well as patient experiences in ED and on the paediatric ward.

Methods An electronic survey, alongside face-to-face discussion, was used to ascertain staff confidence levels, prior training and potential ideas for innovation in paediatric pain management. Alongside this a paper survey was sent to 50 families whose children were either seen in ED or admitted to the ward with a fracture. The feedback was analysed in a focus group involving medical and play specialist staff. We plotted the course of the patient journey constructing a driver diagram.

Results The primary drivers included medicines provision, training and ability and a reassessment process. This culminated in change ideas such as: access to a distraction box in ED, increased awareness of play specialist availability, formal training in both departments and the development of a pain pathway tool.

Conclusion Amongst other systems changes and wider education advances an acute paediatric pain tool was developed. This tool has been designed to be used initially at triage and then throughout an admission. The tool includes pain scores and guidance on pain management depending on the score and injury pattern. This tool has been reviewed at the paediatric clinical governance meeting and we are exploring its introduction aiming to evaluate the effect of the tool on staff confidence and patient experience after six months.

\section{G91(P) CONTACTLESS HEART MONITORING USING MMWAVE RADAR - A RESEARCH STUDY}

${ }^{1}$ RSS Veeravalli, ${ }^{2} \mathrm{ACB}$ Setti, ${ }^{1} \mathrm{~N}$ Dahnoun. ${ }^{1}$ SCEEM (School of Computer Science, Electrical and Electronic Engineering, University of Bristol, Bristol, UK; ${ }^{2}$ Child Health department, Hywel Dda University Health Board, Haverfordwest, UK

\subsection{6/archdischild-2020-rcpch.72}

Introduction Significant burns and skin damage are caused by the probes of the pulse oximetry devices that are used to measure the pulse rates and oxygen saturations in neonates and children. The Royal College's 'Paediatrics 2040' Project aims to look at new innovations and technologies to improve the care of children. Hence we researched one such technology.

Aims To investigate a novel method of 'non-invasive' heart rate monitoring using mmWave radar sensing technology.

Methods To utilise and evaluate Texas Instrument's IWR1443 mmWave radar sensor (similar to radar, but uses short wavelengths for high resolution). A frame of transmitted signals, 
Tx, is used to measure the velocity of two. An IF signal is calculated by mixing the transmitted and received signals. Each IF signal undergoes an FFT to find a peak corresponding to the range. A second FFT transforms the set of RangeFFT results so that two objects can be distinguished by their phase displacements, which correspond to their different velocities. Therefore, the heart rate can be distinguished from the breathing rate. (a) A frame of transmitted signals are used to (b) detect the range of the chest, which can be (c) transformed into heart rate and breathing rate using FFTs. The IWR1443 mmWave radar sensor obtains heart rate measurements by detecting miniscule movements of the chest caused by the heart's pulse and breathing.

Results A Graphical User Interface was developed using C and MATLAB to display a cardiac waveform and a graph of heart rate values in apparent real-time. Experiments using moving averages were performed to increase the accuracy of the sensor's values.

Conclusion This relatively new mmWave radar technology can be used fairly accurately for the pulse rate monitoring in neonates, children, burns patients or for sleep studies. Being contactless, it prevents skin damage and burns. We recommend the 'Paediatrics 2040' project to encourage the Use and further Research of such monitoring devices to decrease risks.

\section{G92(P) INTER-PROFESSIONAL TRIPARTITE ALLIANCE TO REDUCE MEDICATION ERRORS IN CHILDREN}

${ }^{1} \mathrm{D}$ Paccagnella, ${ }^{2} \mathrm{R}$ Issac, ${ }^{3} \mathrm{~B}$ Patel, ${ }^{1} \mathrm{P}$ Vallabhaneni. 'Paediatrics Department, Swansea Bay University Health Board, Morriston Hospital, Swansea, UK; ${ }^{2}$ Nursing and Midwifery Department, Swansea Bay University Health Board, Morriston Hospital, Swansea, UK; ${ }^{3}$ Pharmacy Department, Swansea Bay University Health Board, Morriston Hospital, Swansea, UK

\subsection{6/archdischild-2020-rcpch.73}

Aims Healthcare professionals are faced with a number of challenges when prescribing and administering medication to children, due to the complexities of age-related drug calculations. Internationally, multiple studies have shown that the regular occurrence of such errors carries a high potential for unintended harm to patients, with research suggesting that good quality care depends upon different professions working together.

In light of this, we reasoned that reducing medication errors could be achieved by multidisciplinary education. For this purpose, a paediatric tripartite alliance between Medicine, Nursing and Pharmacology was created.

Methods An initial prospective audit was undertaken, with the aim of recording the number, nature and severity of reported medication errors over a three - month period. Errors were identified using the Datix database and were classified according to the EQUIP study model.

Following the initial audit, a structured educational programme was introduced to all medical and nursing staff. Subsequently, data was collected over two separate three-month periods.

Results During the pre-intervention period, a total of 159 children were admitted to the paediatric inpatient wards. The total number of recorded medication errors was 142 .

During the first post-intervention period, a total of 470 children were admitted and 57 errors were recorded. Compared with the pre-intervention data, where $89.3 \%$ of admitted patients had been subjected to errors, this value decreased to $12.1 \%$, with an estimated error reduction of $77.2 \%$.

During the second post-intervention period, a total of 338 patients were admitted, with 120 errors recorded (35.5\%).

Conclusions The commitment to tackle the issue of medication errors as part of an inter-professional tripartite alliance, coupled with the implementation of a structured programme, have contributed to a significant reduction in medication errors.

Further improvement could be achieved by understanding and targeting specific environmental and human factors, and by addressing any unmet training needs.

We believe that implementation of similar educational programmes on a wider scale would be achievable and costeffective.

\section{G93(P) FOLLOW UP SKELETAL SURVEY IN CHILD PROTECTION MEDICALS: A QUALITY IMPROVEMENT PROJECT}

L Shipley, A Omokanye, V Walker. Community Paediatrics, Sherwood Forest NHS Trust, Sutton-in-Ashfield, UK

\subsection{6/archdischild-2020-rcpch.74}

Introduction Skeletal fractures are estimated to occur in half of children who have suffered physical abuse. ${ }^{1}$ Fractures secondary to non-accidental injury are often occult and risk under detection if single skeletal survey is undertaken; hence follow up is recommended at 11 to 14 days. $^{2}$ Following a serious case review, quality improvement (QI) service changes were implemented aimed at standardising and simplifying logistics, engaging parents/social services at initial survey, reducing reliance on on-call teams and improving communication to reduce human and system errors.

We evaluated the number of patients who met national criteria for follow up skeletal survey and timing of follow up, if undertaken, both pre and post QI changes.

Method Retrospective audit of children undergoing follow up skeletal survey comparing the rate of follow up within 11-14 days and up to 28 days between June 2017 and June 2019. Data were collected from electronic hospital records and case notes and analysed using Microsoft Excel and Stata with significance as $\mathrm{p}<0.05$.

Results 53 children were included in the audit (20 prechange and 33 post change). QI changes to provision of service resulted in significantly less children missing follow up skeletal survey $(45 \% \quad(n=9) \quad$ vs $3 \% \quad(n=1), p=0.002)$. $68.7 \%(n=22)$ of follow up surveys occurred within the gold standard of 11 to 14 days following QI change compared to $45 \% \quad(n=5)$ prior. Of children who underwent skeletal survey $45 \%$ presented with bruising and 17\% with fracture.

Conclusion Follow-up skeletal survey is an essential part of the child protection medical pathway, occurring ideally within 11 to 14 days, to optimally detect healing fractures. The following implemented changes have led to valuable learning which provides opportunity for change to other teams:

- Booking x-ray at time of first skeletal survey

- Radiology service responsible for making appointment

- Clear discussion and expectation of social worker and carers to attend second appointment 